\title{
Defect Turbulence in Inclined Layer Convection
}

\author{
Karen E. Daniels and Eberhard Bodenschatz* \\ Laboratory of Atomic and Solid State Physics, Cornell University, \\ Ithaca, New York 14853
}

(Received 5 July 2001; published 7 January 2002)

\begin{abstract}
We report experimental results on the defect turbulent state of undulation chaos in inclined layer convection of a fluid with Prandtl number $\approx 1$. By measuring defect density and undulation wave number, we find that the onset of undulation chaos coincides with the theoretically predicted onset for stable, stationary undulations. At stronger driving, we observe a competition between ordered undulations and undulation chaos, suggesting bistability between a fixed-point attractor and spatiotemporal chaos. In the defect turbulent regime, we measured the defect creation, annihilation, entering, leaving, and rates. We derive a universal probability distribution function which agrees with the experimental findings.
\end{abstract}

PACS numbers: 47.54.+r, 47.20.Bp

Weakly driven, dissipative pattern-forming systems often exhibit the spatiotemporally chaotic state of defect turbulence, where the dynamics of a pattern is dominated by the perpetual nucleation, motion, and annihilation of point defects (or dislocations) [1]. Examples can be found within striped patterns in wind driven sand, electroconvection in liquid crystals [2], nonlinear optics [3], fluid convection [4,5], autocatalytic chemical reactions [6], and Langmuir circulation in the oceans [7]. The hope is that the dynamics of these very different systems can be characterized by a universal description which is based only on the defect dynamics.

A first description of defect turbulence was given by Gil et al. [8] for a spatiotemporally chaotic state of the complex Ginzburg-Landau equation (CGLE). They postulated that the nucleation rate for defect pairs is independent of the number of pairs $M$, and based on the topological nature of defects the annihilation rate is proportional to $M^{2}$. Through detailed balance, they showed that these assumptions lead to a squared Poisson distribution for the probability distribution function (PDF) of $M$. They also found agreement with this PDF in simulations of the CGLE with periodic boundary conditions. Rehberg et al. [2] measured the PDF of $M$ for defect turbulence in electroconvection of nematic liquid crystals and found it to be consistent with the predicted squared Poisson distribution. Later, Ramazza et al. [3] investigated a defect turbulent state in optical patterns and found that their data were not conclusive. To date, studies in both simulation and experiment have relied purely on comparisons of the PDFs. The gain and loss rates, fundamental to the universal description of defect turbulence, have not been measured. In addition, effects due to boundaries were not considered.

In this Letter, we report experimental results on the defect turbulent state of undulation chaos in inclined layer convection of a fluid of Prandtl number $\approx 1$. By tracking all defects in a finite area of the convection cell we measured, for the first time, defect creation, annihilation, leaving, and entering rates for a defect turbulent state. The observed pair creation and annihilation rates agree with the predictions [8]. In an experimental system where periodic boundary conditions do not apply, pairs of defects are no longer an appropriate description. To describe the statistics correctly, single defect leaving and entering rates through the boundaries must be considered. Our data show that the entering rates are approximately independent of the number of positive/negative defects $N_{ \pm}$and the leaving rates are proportional to $N_{ \pm}$. We derive a universal PDF which reduces to the earlier predicted squared Poisson distribution [8] when boundary effects are negligible. Our measurements agree with this new distribution. In addition, by measuring both $N_{ \pm}$and the undulation wave number we determined that the onset of undulation chaos coincides with the theoretically predicted onset for stable, stationary undulations. At higher driving, we observe competition between regions of ordered undulations and undulation chaos, suggesting bistability between a fixed-point attractor and spatiotemporal chaos.

When an inclined fluid layer (see Fig. 1) is heated from below and cooled from above for small temperature differences $\Delta T$ the fluid layer experiences not only a linear temperature gradient (as in Rayleigh-Bénard convection) but also a symmetry-breaking large scale shear flow, with fluid rising at the warmer plate and falling at the cooler plate [9]. Above a critical temperature difference $\Delta T_{c}$, a pattern of longitudinal rolls aligned with the uphill/downhill direction is observed for intermediate inclination angles. For $\epsilon \gtrsim 0.02$, where $\epsilon \equiv \Delta T / \Delta T_{c}-1$, these longitudinal

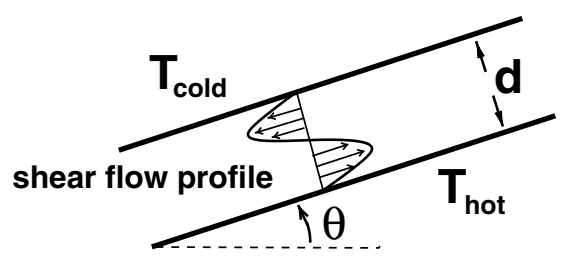

FIG. 1. Schematic drawing of inclined layer convection of cell thickness $d$ and temperature difference $\Delta T \equiv T_{\text {hot }}-T_{\text {cold }}$. 
rolls are unstable to undulations $[9,10]$. While it was predicted theoretically that a perfect pattern of undulations is stable [11], a defect turbulent state of undulation chaos was found experimentally [12]. Recently, undulation chaos was also observed in numerical simulations of the NavierStokes equations when random initial conditions were used [11], while with controlled initial conditions undulations were found to be stable. As shown in the snapshot in Fig. 2a, undulation chaos is characterized by a pattern of disordered undulating rolls, punctuated by point defects which carry a topological charge of $\pm 2 \pi$ [15]. Defects are nucleated pairwise in regions of low convective amplitude (green), enter and leave the system at the boundaries, preferentially move at right angles to the rolls, and annihilate as pairs. For $\epsilon \geq 0.10$, the defect turbulent state appears intermittently with ordered regions of undulations, suggesting a competition between the fixed-point attractor (undulations) and spatiotemporal chaos (undulation chaos). This is shown by the two snapshots in Figs. $2 b$ and $2 c$. The dynamics of the state is best viewed in the movies available at Ref. [13].

The experiments were conducted in the same apparatus as in Ref. [12] for an inclination angle of $\theta=30^{\circ}$. The fluid was compressed $\mathrm{CO}_{2}$ at a pressure of $(56.5 \pm$ $0.01)$ bar regulated to \pm 0.005 bar with a mean temperature of $(28 \pm 0.05){ }^{\circ} \mathrm{C}$ regulated to $\pm 0.0003{ }^{\circ} \mathrm{C}$. For these parameters, convection appeared at $\Delta T_{\mathrm{c}}=(1.763 \pm$ $0.005){ }^{\circ} \mathrm{C}$. The planform of the convection pattern was observed by the usual shadowgraph technique [16]. A cell of height $d=(388 \pm 2) \mu \mathrm{m}$ and dimensions $101 d \times$ $50 d$ was used, for which the vertical diffusion time was $\tau_{v} \approx 1.3 \mathrm{sec}$. All data were measured in a homogeneous central subregion of size $32 d \times 25 d$ [17]. To track defects, runs of 100 shadowgraph images were taken at a rate of 3 frames $/ \sec \left(\approx \tau_{v} / 4\right)$ with a $1008 \times 1018$ pixel digital CCD camera. For 17 values of $\epsilon$ between 0.04 and 0.22 this was repeated for $600(500,400)$ runs at $\epsilon \leq 0.07(0.08 \leq \epsilon \leq 0.10, \epsilon \geq 0.12)$. Runs at the same $\epsilon$ were separated by at least $100 \tau_{v}$ for statistical inde-

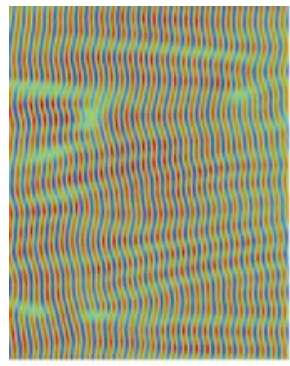

(a)

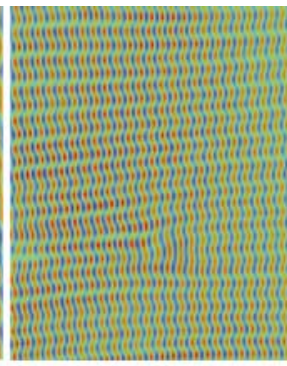

(b)

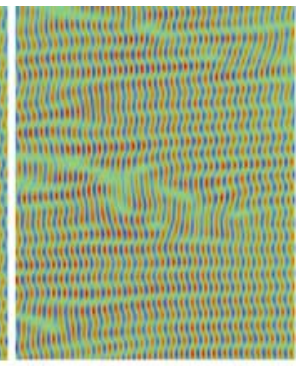

(c)
FIG. 2 (color). False color images of undulation chaos [13] at $\theta=30^{\circ}$. Red/blue is the rising/falling fluid and green is the low-amplitude convection. The uphill direction is at the top of the page, and the region shown is the subregion of the cell used in the analysis. Positive defects point uphill, negative defects downhill, and the pattern drifts downward [14] with a period $\approx 150 \tau_{v}$. (a) $\epsilon=0.08$; (b) $\epsilon=0.17$; (c) $\epsilon=0.17$. pendence. A total of 1.5 terabytes of data were analyzed. Each value of $\epsilon$ was reached via a quasistatic temperature increase in steps of $0.001{ }^{\circ} \mathrm{C}$, waiting $700 \tau_{v}$ between steps for transients to die out. We also conducted a sequence of measurements with quasistatic temperature decreases to check for possible hysteresis, which was not observed.

To lowest order, the planform of undulation chaos can be captured by the real part of the two-dimensional field $\Psi(x, y)$ with

$$
\Psi=\psi_{0} e^{i(q x+\phi)}+i \psi_{1}\left(e^{i(q x+p y)}+e^{i(q x-p y)}\right)+\ldots,
$$

where the wave numbers $q$ and $p$ describe the stripe spacing and waviness, respectively. Both the wave numbers and the amplitudes $\psi_{0}$ and $\psi_{1}$ vary slowly in space and time. For a perfect undulation pattern, $\phi$ is constant everywhere, while, for undulation chaos, $\phi(x, y, t)$ approximates the deformation of the pattern due to the defects. The ansatz $\phi(x, y, t)=\sum c_{i} \arctan \left(\frac{y-y_{i}}{x-x_{i}}\right)$ describes the observed pattern well for constant $q, p$, and $\psi_{1} / \psi_{0}$, where $\left(x_{i}, y_{i}\right)$ are the locations of defects and $c_{i}= \pm 1$ are their corresponding topological charges.

In Fig. 3 the peak values for the distributions of $p, q$, and $\psi_{1} / \psi_{0}$ are shown as a function of $\epsilon$ [18]. The values for $q$ change little with $\epsilon$, while the undulation wave numbers can be fit by $p \propto \sqrt{\epsilon-\epsilon_{u}}$, shown as a solid black line. This fit provides a value for the onset of undulation chaos at $\epsilon_{u}=0.017 \pm 0.001$, which is in good agreement with $\epsilon_{u}=0.016$ predicted theoretically for the onset of stable stationary undulations [11].

In order to better characterize the generic properties of defect turbulence, we determined defect locations and the corresponding topological charges in each shadowgraph image. A time trace of the number of defects over $2 \times 10^{5} \tau_{v}$ at $\epsilon=0.13\left(10^{4}\right.$ defects) revealed that the undulation chaos state is statistically stationary and neutral with respect to topological charge. Nonetheless, any single realization of the pattern does not necessarily satisfy $N_{+}=N_{-}$due to the boundaries. From the list of defect
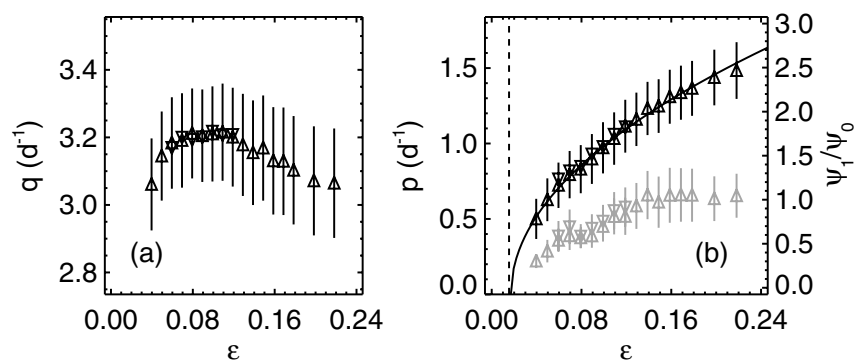

FIG. 3. (a) Roll wave number $q$ and (b) undulation wave number $p$ (black) with relative amplitude $\psi_{1} / \psi_{0}$ (gray), as a function of $\epsilon$. Values are taken at the peaks of the PDF, with bars representing the width of the distribution at one standard deviation. Data taken while increasing the temperature are shown with symbol $\triangle$ and, while decreasing the temperature, with $\nabla$. The dashed line represents the prediction for the onset from Ref. [11]. 
locations in each frame, we determined the mean $\left\langle N_{ \pm}\right\rangle$ and the variance $\sigma_{ \pm}^{2}=\left\langle N_{ \pm}^{2}\right\rangle-\left\langle N_{ \pm}\right\rangle^{2}$ in the chosen subregion, plotted in Fig. 4 as a function of $\epsilon$. Here and below, we report results only for $N_{+}$; equivalent results were obtained for $N_{-}$.

For $\epsilon \leq 0.10, N_{ \pm}(\epsilon)$ is linearly increasing. Extrapolation to $N_{+}=0$ gives $\epsilon_{d}=0.025 \pm 0.002$, which is close to the onset of undulations $\left(\epsilon_{u}=0.017 \pm 0.001\right)$ determined above from wave number measurements. The difference may be attributable to the finite size of the system. For $\epsilon>0.10, N_{ \pm}(\epsilon)$ decreases in the bistability regime, while no such decrease is seen in $p$.

Two predicted generic features of defect turbulence [8] are the constant creation rate and the annihilation rate quadratic in the number of defect pairs $M$. By connecting defect locations in adjacent frames to defect tracks, and then associating tracks of opposite sign to locate creation and annihilation events, we were able to perform the first direct test of the theoretical postulates, as well as the entering and leaving rates to/from the observation area. For a system where single defects can enter and leave through the boundaries a pair-based description is no longer justified. Thus, we separately consider the PDFs for $N_{ \pm}$.

The creation rate $C$ was observed to be approximately independent of $N_{ \pm}$and the annihilation rate agreed with $A \propto N_{+} N_{-}$, by extension from $A \propto M^{2}$ for the topologically neutral case. Figure 5 shows the four gain/loss rates, tabulated using positive defects and events only. To lowest order, the entering rate is independent of the number of defects already in the subregion of the cell, suggesting that both creation and entering are random processes. The leaving rate was found to increase in proportion to $N_{ \pm}$as expected, since unlike annihilations such events do not depend on the presence of two oppositely charged defects.

The observed rates are approximately given by

$$
\begin{aligned}
& E\left(N_{ \pm}\right)=E_{0}, \\
& C\left(N_{ \pm}\right)=C_{0}, \\
& L\left(N_{ \pm}\right)=L_{0} N_{ \pm}, \\
& A\left(N_{ \pm}\right)=A_{0} N_{ \pm}^{2},
\end{aligned}
$$

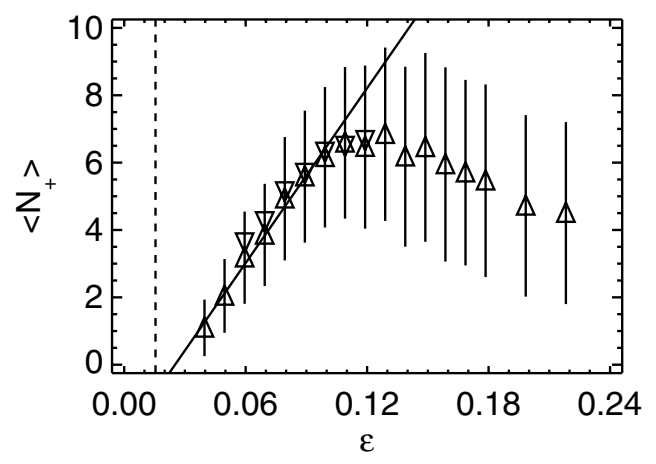

FIG. 4. Number of positive defects in the subregion as a function of $\epsilon$, with bars representing the width of the distribution at one standard deviation. which consider defects of a single sign only. The fits corresponding to Eqs. (2) are shown in Fig. 5, for three values of $\epsilon$. It is important to note that this is a simplification. $C\left(N_{ \pm}\right)$and $E\left(N_{ \pm}\right)$show a weak, negative slope: defects are slightly less likely to enter or be created if there are already defects present. We also observe a weak dependence of these rates on the number of oppositely charged defects: $E\left(N_{+}\right)$is slightly diminished and $L\left(N_{+}\right)$is slightly elevated for $N_{+}>N_{-}$. Thus, to fully describe the system, we would need to consider rates and distributions for both $N_{+}$and $N_{-}$simultaneously. In order to make analytical progress we will assume that Eqs. (2) are a complete description. As will be shown below, these assumptions approximate the data well.

Using Eqs. (2), we can construct the PDF for $N \equiv N_{ \pm}$. Assuming a stationary distribution, detailed balance requires $\operatorname{loss}(N) \mathcal{P}(N)=\operatorname{gain}(N-1) \mathcal{P}(N-1)$ to describe the probabilities at adjacent $N$ [19]. For the relevant rates,

$$
\begin{aligned}
\mathcal{P}(N) & =\frac{E(N)+C(N)}{L(N)+A(N)} \mathcal{P}(N-1) \\
& =\frac{\alpha}{\beta N+N^{2}} \mathcal{P}(N-1) .
\end{aligned}
$$

where $\alpha \equiv \frac{E_{0}+C_{0}}{A_{0}}$ and $\beta \equiv \frac{L_{0}}{A_{0}}$. By performing the recursion and properly normalizing the distribution, we find a modified Poisson distribution

$$
\mathcal{P}_{\alpha, \beta}(N)=\frac{\alpha^{(\beta / 2)+N}}{I_{\beta}(2 \sqrt{\alpha}) \Gamma(1+\beta+N) N !},
$$

where $I_{\beta}$ is the modified Bessel function. For $\beta=0$ $\left(\alpha=\left\langle N^{2}\right\rangle\right)$ this PDF reduces to the squared Poisson distribution of Ref. [8]. In our experiments, $\beta \approx 3$ [20].

Figure $6 \mathrm{~b}$ shows that the squared Poisson and Poisson distributions match the data poorly. Both the modified Poisson distribution and a distribution obtained by performing the recursion of Eq. (3) on the raw experimental
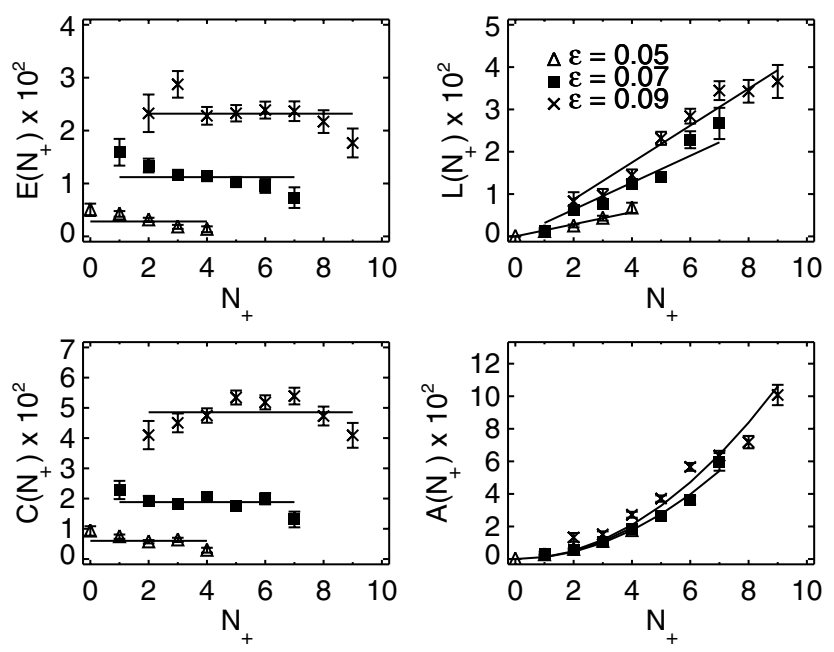

FIG. 5. Probability of entering and of leaving, creation, and annihilation (per $0.33 \mathrm{sec}$ ) as a function of $N_{+}$for several $\epsilon$. Lines are fits to Eq. (2). 


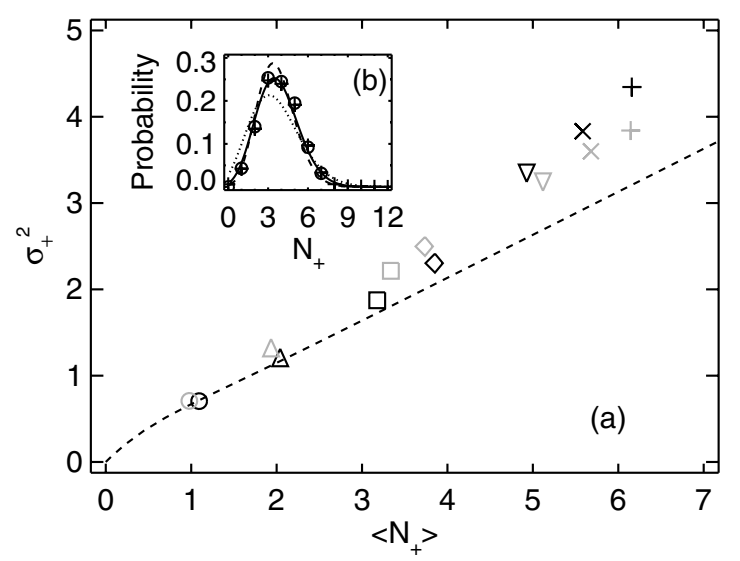

FIG. 6. (a) Mean number of positive defects vs $\sigma_{+}^{2}$ for $\epsilon$ between 0.04 and 0.10 . The black symbols are experimental; the gray symbols are from Eqs. (5) and (6); the dashed line is the analytical relation for squared Poisson distribution [8]. (b) PDF for $6 \times 10^{4}$ defects at $\epsilon=0.07$, with four distributions shown for comparison. + are the experimental distribution, the solid line is modified Poisson, the dashed line is squared Poisson, the dotted line is Poisson, and $\bigcirc$ are from the raw rates in Fig. 5.

data from Fig. 5 agree well with the experimental data. The modified Poisson distribution also correctly captures the mean and width of the PDF. Using the already normalized modified Poisson distribution [Eq. (4)], we calculate $\langle N\rangle=\sum N \mathcal{P}_{\alpha, \beta}(N)$ and $\left\langle N^{2}\right\rangle=\sum N^{2} \mathcal{P}_{\alpha, \beta}(N)$ and obtain the analytic expressions

$$
\begin{gathered}
\langle N\rangle=\sqrt{\alpha} \frac{I_{\beta+1}(2 \sqrt{\alpha})}{I_{\beta}(2 \sqrt{\alpha})}, \\
\left\langle N^{2}\right\rangle=\sqrt{\alpha} \frac{I_{\beta+1}(2 \sqrt{\alpha})+\sqrt{\alpha} I_{\beta+2}(2 \sqrt{\alpha})}{I_{\beta}(2 \sqrt{\alpha})} .
\end{gathered}
$$

Again, the above equations reduce to the corresponding equations for the squared Poisson distribution in the limit $\beta=0$. In Fig. 6a the dependence of the mean on the variance is plotted for the raw data and the modified and squared Poisson distributions. The modified Poisson distribution [Eq. (6)] shows excellent agreement, while the squared Poisson distribution clearly fails to describe the experimental data.

In summary, we find that the state of undulation chaos, and not the fixed-point attractor to ordered undulations, is selected above the predicted onset for undulations. At higher driving, we find an apparent competition between the two attractors.

Our result for the $N$ dependence of the defect creation, annihilation, leaving, and entering rates and the modified Poisson [Eq. (4)] should be generic to any defect turbulent system. We have found agreement with the theoretical predictions for creation and annihilation rates [8] and have extended the analysis to the experimentally relevant case of a finite system without periodic boundary condi- tions, where it is important to consider both positive and negative defects (rather than pairs). Although we observed weak fluctuations away from topological charge neutrality, a description based purely on a single topological charge was sufficient to describe the observed behavior.

We thank W. Pesch and O. Brausch for sharing the results of simulations and stability analysis which have enhanced our understanding of the bistability. We also thank the NSF for support under DMR-0072077.

*Electronic address: eb22@cornell.edu

[1] P. Coullet, L. Gil, and J. Lega, Phys. Rev. Lett. 62, 1619 (1989).

[2] I. Rehberg, S. Rasenat, and V. Steinberg, Phys. Rev. Lett. 62, 756 (1989).

[3] P. Ramazza, S. Residori, G. Giacomelli, and F. Arecchi, Europhys. Lett. 19, 475 (1992).

[4] S. W. Morris, E. Bodenschatz, D. S. Cannell, and G. Ahlers, Phys. Rev. Lett. 71, 2026 (1993).

[5] A. L. Porta and C. M. Surko, Physica (Amsterdam) 139D, 177 (2000).

[6] Q. Ouyang and J. M. Flesselles, Nature (London) 379, 143 (1996).

[7] T. M. Haeusser and S. Leibovich, Phys. Rev. Lett. 79, 329 (1997).

[8] L. Gil, J. Lega, and J. L. Meunier, Phys. Rev. A 41, 1138 (1990).

[9] J. E. Hart, J. Fluid Mech. 47, 547 (1971).

[10] R. M. Clever and F.H. Busse, J. Fluid Mech. 81, 107 (1977).

[11] W. Pesch and O. Brausch (unpublished); O. Brausch, Ph.D. thesis, University of Bayreuth, 2001.

[12] K. E. Daniels, B. B. Plapp, and E. Bodenschatz, Phys. Rev. Lett. 84, 5320 (2000).

[13] MPEG movies of undulation chaos are available at http:// milou.msc.cornell.edu/ILCmovies/ and see AIP Document No. EPAPS: E-PRLTAO-88-001203 for mpeg movies of Fig. 1. This document may be retrieved via the EPAPS homepage (http://www.aip.org/pubservs/epaps.html) or from ftp.aip.org in the directory /epaps/. See the EPAPS homepage for more information.

[14] Drifting is due to weak non-Boussinesq effects, as with all transverse modes of inclined layer convection [12].

[15] M. C. Cross and P. C. Hohenberg, Rev. Mod. Phys. 65, 851 (1993).

[16] J. R. de Bruyn et al., Rev. Sci. Instrum. 67, 2043 (1996).

[17] This was the largest region which showed random defect tracks. For smaller observation areas, the statistics behaved equivalently.

[18] Local wave numbers were measured via a method similar to Ref. [21] and amplitudes were measured locally via a method to be published.

[19] Analysis of time-reversed raw data reveals that the time symmetry required for detailed balance is satisfied.

[20] For large $N_{ \pm}$, the PDF becomes Gaussian and the underlying mechanisms are obscured.

[21] D. A. Egolf, I. V. Melnikov, and E. Bodenschatz, Phys. Rev. Lett. 80, 3228 (1998). 